\title{
Interrogating Social Virtual Reality as a Communication Medium for Older Adults
}

\author{
STEVEN BAKER, The University of Melbourne, Australia \\ RYAN M. KELLY, The University of Melbourne, Australia \\ JENNY WAYCOTT, The University of Melbourne, Australia \\ ROMINA CARRASCO, The University of Melbourne, Australia \\ THUONG HOANG, Deakin University, Australia \\ FRANCES BATCHELOR, National Ageing Research Institute, Australia \\ ELIZABETH OZANNE, The University of Melbourne, Australia \\ BRIONY DOW, National Ageing Research Institute, Australia \\ JENNIFER WARBURTON, La Trobe University, Australia \\ FRANK VETERE, The University of Melbourne, Australia
}

A growing body of research is examining the way that virtual reality (VR) technology might enrich the lives of older adults. However, no studies have yet examined how this technology-combining head mounted displays, motion tracking, avatars, and virtual environments-might contribute to older adult wellbeing by facilitating greater social participation (social VR). To address this gap, we conducted three workshops in which 25 older adults aged 70 to 81 explored the utility of social VR as a medium for communicating with other older adults. Participants first created embodied avatars that were controlled through natural gestures, and subsequently used these avatars in two high-fidelity social VR prototypes. Findings from the workshops provide insight into older adults' design motivations when creating embodied avatars for social VR; their acceptance of social VR as a communication tool; and their views on how social VR might play a beneficial role in their lives Outcomes from the workshops also illustrate the critical importance our participants placed on behavioural anthropomorphism-the embodied avatars' ability to speak, move, and act in a human-like manner- alongside translational factors, which encapsulate issues relating to the way physical movements are mapped to the embodied avatar and the way in which errors in these mappings may invoke ageing stereotypes. Findings demonstrate the critical role that these characteristics might play in the success of future social VR applications targeting older users. We translate our findings into a set of design considerations for developing social VR systems for older adults, and we reflect on how our participants' experiences can inform future research on social virtual reality.

CCS Concepts: • Human-centered computing $\rightarrow$ Virtual reality; Collaborative and social computing; Empirical studies in collaborative and social computing.

Additional Key Words and Phrases: Older adults, virtual reality, avatars, communication.

Authors' addresses: Steven Baker, The University of Melbourne, Parkville, Melbourne, VIC, 3010, Australia, steven.baker@ unimelb.edu.au; Ryan M. Kelly, The University of Melbourne, Parkville, Melbourne, VIC, 3010, Australia, ryan.kelly@unimelb. edu.au; Jenny Waycott, The University of Melbourne, Parkville, Melbourne, VIC, 3010, Australia, jwaycott@unimelb.edu.au; Romina Carrasco, The University of Melbourne, Parkville, Melbourne, VIC, 3010, Australia, rcarrasco@student.unimelb.edu. au; Thuong Hoang, Deakin University, Geelong, VIC, 3220, Australia, thuong.hoang@deakin.edu.au; Frances Batchelor, National Ageing Research Institute, Parkville, Melbourne, VIC, 3010, Australia, F.Batchelor@nari.edu.au; Elizabeth Ozanne, The University of Melbourne, Parkville, Melbourne, VIC, 3010, Australia, eao@unimelb.edu.au; Briony Dow, National Ageing Research Institute, Parkville, Melbourne, VIC, 3010, Australia, b.dow@nari.edu.au; Jennifer Warburton, La Trobe University, Wodonga, VIC, 3690, Australia, J.Warburton@latrobe.edu.au; Frank Vetere, The University of Melbourne, Parkville, Melbourne, VIC, 3010, Australia, f.vetere@unimelb.edu.au.

(C) 2019 Copyright held by the owner/author(s). Publication rights licensed to ACM.

This is the author's version of the work. It is posted here for your personal use. Not for redistribution. The definitive Version of Record was published in Proceedings of the ACM on Human-Computer Interaction, https://doi.org/10.1145/3359251. 


\section{ACM Reference Format:}

Steven Baker, Ryan M. Kelly, Jenny Waycott, Romina Carrasco, Thuong Hoang, Frances Batchelor, Elizabeth Ozanne, Briony Dow, Jennifer Warburton, and Frank Vetere. 2019. Interrogating Social Virtual Reality as a Communication Medium for Older Adults. Proc. ACM Hum.-Comput. Interact. 3, CSCW, Article 149 (November 2019), 24 pages. https://doi.org/10.1145/3359251

\section{INTRODUCTION}

Virtual Reality (VR) technology has been the subject of intense academic interest since the late 1960 s, but it is only recently that VR has gained traction as a mass market consumer technology. This resurgence has been accompanied by a renewed interest in the potential for VR to have a positive impact on the lives of older adults. A growing body of research has examined how the immersive properties of VR might be used to assist older adults in areas such as recovery after a major illness [32, 67], exercise [48, 67], responding to the impacts of dementia [30, 49], and as a tool for encouraging reminiscence [8]. However, to date, the potential for VR to be used as a social communication platform (social VR) by older adults has yet to be explored. Social VR has the potential to introduce new opportunities for social participation among older adults in virtual space, going beyond the opportunities enabled by existing technologies such as video calls or online social networks. Indeed, recent research has indicated that older adults are interested in the potential for 3D virtual environments to support social experiences [e.g. 65], but no research has yet explored older adults' preferences within the context of immersive social VR technologies.

In this paper we address this gap by conducting a study involving 25 older adults aged 70-81, who participated in three workshops that allowed them to experience core aspects of social VR. In the first workshop they designed embodied avatars - digital self-representations that are controlled via natural body movements and gestures [53]. In the second and third workshops, these avatars were used in two different social VR prototypes, where participants interacted with each other via voice while controlling their avatars. We use the workshops to investigate older adults' acceptance of VR as a tool for social communication and the ways in which our participants saw social VR as potentially beneficial for the lives of older adults. These workshops also allow us to examine, for the first time, older adults' views about the design and use of embodied avatars in a social virtual world.

Participants were enthusiastic about the potential of social VR as a tool for enriching their own lives and the lives of other older adults, particularly those who are socially isolated. Our findings highlight the importance older adults place on avatar anthropomorphism - the embodied avatars' ability to speak move and act in "ways that might be expected of humans" [53, p.47]. Our study also sheds light on the importance placed by older adults on the way body movements are translated onto embodied avatars in the virtual environment and the impact that tracking errors can play on perpetuating negative ageing stereotypes. To emphasise the importance of this finding, we contribute an extension to an existing framework and argue that these Translational factors should be combined with User and Platform factors when assessing the quality of social interactions experienced by older adults in social VR [65]. We conclude with guidelines to support the design of future social VR applications that are more suited to the needs of older adults.

\section{RELATED WORK}

\subsection{Older Adults, ICTs and Social Participation}

There has been a long standing focus within the HCI and CSCW communities on the role that technology can play in responding to the needs of older adults. Research suggests that older people are very accepting of digital technologies, provided that the technologies in question are highly usable and have demonstrable value for enhancing people's lives [e.g. 78]. It has also been recognised 
that technologies should be designed to accommodate the physical and cognitive changes that arise as people grow older, such as by designing more intuitive user interfaces [25, 35, 52].

Other research has sought to address the broader challenge of supporting older adults in living healthier lives and ageing 'in place' [36, 40,56]. A key element of this research has been the design and evaluation of technologies that seek to address social isolation and/or encourage social participation [6], given that social disconnectedness is a risk factor that is known to impact older adults' health and wellbeing $[20,24]$. Prior work has shown how communication technologies can address social disconnectedness by, for example, mediating conversation between grandparents and their grandchildren [26] or by enabling intergenerational play over distance [29, 73].

A related body of work has examined older adults' use of social media. A number of issues are particularly important to older users, including concerns about privacy and anonymity [13, 31, 47] and a desire for interfaces that are easy-to-use [18, 41]. Although the use of online communication platforms by older adults is increasing, research suggests that those over the age of 70 are often reluctant to embrace online communication platforms because of the way in which these systems are designed to promote lightweight interactions [31, 41]. In considering future goals for technologies that address older adults' reluctance to embrace social media, Hope and colleagues have shown that older adults value "deeper, well thought out, carefully crafted social communications" and modalities that "enable depth of thought, reflection, and personalization" [31, p.3911]. Other research has similarly demonstrated the importance of these so called 'heavyweight' communications to older adults [31, 41], emphasising the need to consider how technologies can allow older adults to remain in touch with other people in ways that are more meaningful and acceptable to them [26, 41, 52].

To this end, Brewer and Piper [11] conducted in-depth interviews with 20 older adult bloggers and developed a number of recommendations for designing for late-life development. Chief amongst these was the need to support "multiple, changing identities in older adulthood" by designing online systems that "support complex and multi-faceted identities of older adults" [11, p.9]. Their work is reflective of a broader movement within $\mathrm{HCI}$ that emphasises the capabilities and heterogeneity of older adults over deficits and pejorative ageing stereotypes [2, 3, 74].

HCI research with older adults embraces new and emerging technology as a means of encouraging social participation. Touch-screen interfaces, in particular, have been applied across a range of domains, often in combination with bespoke user interfaces that employ a simplified design to enable the sharing of digital artefacts $[6,7,52,75]$. Exergames have also received some attention in the literature $[16,21,71]$. Though research has focused predominantly on the impacts of exergames on physical health, their use of natural body gestures to control embodied avatars in games has been noted to have positive social effects [16, 71]. This suggests opportunities for social participation that are quite different to previous modalities.

Social VR is an example of an emerging consumer technology that has potential as a communication medium. By incorporating immersive experiences and interaction via natural gestures, social VR could provide older adults with new opportunities to engage in meaningful social interactions by meeting other users in virtual space [7]. The use of embodied avatars in social VR likewise presents opportunities to address Brewer and Piper's call for communication platforms that support self-expression and embrace complex, multifaceted older adult identities [11]. Avatars can also address older adults' concerns for privacy and anonymity by allowing them to choose how they wish to be viewed by others [37]. However, despite authors acknowledging that social VR "will vastly expand" social media opportunities [59, p.128], there has been no research evaluating immersive social VR as a communication medium for older adults. Our work addresses this gap by exploring the emergent needs and concerns of older adults in order to inform the design of future social VR technologies with this population. 


\subsection{Virtual Reality and Older Adults}

In contrast to the lack of research examining social VR for older adults, there has been considerable interest in the application of VR to specific challenges facing older people, for instance as a tool to assist with rehabilitation $[32,48,60,67]$. Key areas of evaluation relate to the use of VR and exergames as tools to improve range of movement [48], and the application of VR to specific conditions such as Parkinson's disease [32]. There has also been interest in the application of VR for individuals living with dementia [30, 43, 49]. For example, Hodge et al. [30], report on a series of design workshops that explored the potential to create virtual reality environments for use by people with dementia and their carers. Though their work was not geared towards designing social VR experiences, they found that participants "saw virtual reality as an opportunity to be together in meaningful ways with their loved ones" by enabling social interaction within the confines of the virtual environment [30, p.8]. These studies illustrate that VR technology has the potential to be acceptable to older adults, but they have not explored the ways in which VR systems might be designed to support greater social participation.

In the wider literature on virtual environments, research has described how online games such as World of Warcraft and open-ended worlds such as Second Life can allow people to socialise online $[12,22,50,58]$. Second Life in particular has been studied in terms of its potential to enhance the lives of older adults by enabling healthcare delivery [19] and online education [61]. Other studies suggest that older adults are interested in using Second Life because it allows them to keep in touch with friends and family $[58,63,64]$ and because it affords opportunities for building new friendships [54] while offering close control over self-disclosure of physical appearance [66]. Older adults also see potential for environments like Second Life to be used for collaborative activities such as online shopping [65] and to address social isolation among those who are housebound [19]. However, work in this space to date has only considered desktop-based systems and has not explored older adults' use of fully immersive VR systems that involve head-mounted displays and other advanced technologies. Our work addresses this gap by exploring the extent to which older adults are accepting of immersive social VR, and explores how they envision this medium being used for social interaction with other adult users.

\subsection{Older Adults and Avatars}

The vast majority of online virtual environments employ the use of avatars as a way of representing users within digital space. An avatar is "a digital representation of a human user that facilitates interaction with other users, entities, or the environment" [53, p.34]. Creating an avatar is a common first step in many virtual worlds [44] and avatar design plays a central role in the development of users' identities within these spaces [46]. While users often create avatars that reflect their own appearance and identity [72], avatars also allow users to explore alternate and imagined identities [9]. However, research has noted that users' avatar design choices are shaped and constrained by the limitations of the software afforded to them [45] and that users typically create avatars that are targeted towards the context in which the avatar is anticipated to be used [44].

In terms of older adults, avatar-based research has predominantly focused on the evaluation of static avatar designs or avatars used in the context of gaming [15, 17], as opposed to customdesigned 3D avatars intended for use in social VR. For example, one exploratory study by Cheong et al. [17] introduced a range of custom-designed static avatars which were simply printed on paper and shown to 24 adults over the age of 55 . More recently, researchers have sought to understand how older adults' avatar designs reflect aspects of their personality and stereotypical views of older adults $[14,15]$. Reed and Fitzpatrick [58] studied the avatars designed by older adults in Second Life, finding that their participants were concerned about self-presentation and did not wish to 
appear 'older' than they really were. Carrasco et al. [15] conducted interviews with ten older adult gamers between the ages of 65 to 95 years. Their findings indicated older adult gamers "designed their player self-representations to project aspects of their lost (former) sel[ves] and to embrace their present older selves" [p.441]. Despite the advantages of avatars in aiding communication [53] and the clear implication that avatars can be used to support approaches to design that embrace "multiple, changing identities in older adulthood" [11, p.9], no studies have attempted to understand older adults' design motivations and use of avatars in social VR situations. Our work addresses this by studying what motivated older adults when they designed their avatars and their reactions to the use of these avatars in two social VR environments.

\section{METHODS}

We conducted three exploratory workshops to gain insight into older adults' perspectives on the potential for social VR to be used as a communication tool. In the first workshop, older adults were invited to design their own embodied avatars. In the second and third workshops, the older adults used these avatars in two different social VR prototypes, each of which was designed to explore a different aspect of the social VR experience.

The first prototype allowed participants to come face-to-face with their own embodied avatar alongside those of their fellow participants. This avatar as third person approach, allowed us to gather the participants' insights into how their personal avatars reacted to their body movements, an approach which is difficult to measure when embodying an avatar from a first person perspective using a head-mounted display (HMD). Each participant was able to control their avatar using natural gestures, i.e. hand and body movements. This was conceived as a means of familiarising the participants with how embodied avatars can respond to gestural inputs as well as giving them a chance to communicate with others 'through' their avatar.

The second prototype involved the use of HMDs, which gave participants the opportunity to use a fully immersive custom-designed VR environment. This prototype also allowed the older adults to experience a virtual world through the eyes of their embodied avatar, i.e. from a first-person perspective, allowing the participants to focus more on the social interactions possible in social VR and their fellow users, rather than the movement of their embodied avatar.

Methodologically, our study is grounded in a critical realist stance that seeks to understand the "causal mechanisms" that underpin experiences of the world [51, p.109]. This stance is most evident in our decision to use a participatory action research (PAR) methodology. Given the prevalence of participatory design and other co-design based methods in $\mathrm{HCI}$ and CSCW, it is important to emphasise that PAR is not an approach to co-design and thus our participants did not actively participate in the creation of the social VR prototypes or the avatar design software. Rather, PAR is a methodology that underpins and guides our entire research process. Key distinguishing features of PAR are its adoption of iterative cycles of planning, action and reflection throughout the research, and its focus on emancipation and empowerment of the participants taking part in the research project [57]. To reflect these features, we designed our workshops to build on one another, and our findings give special privilege to the views of the participants, adopting an approach to research in which older adults are active agents who play a more central role in "establishing the HCI research agenda" [74, p.3]. These qualities are described further in relevant sections below.

\subsection{Participants}

Following the receipt of university ethics approval for the research, we recruited participants by distributing posters advertising the project at various public forums targeting older adults, and in public spaces such as libraries and community notice boards. Participants were required to be 70 years of age and over, be able to speak English to a reasonable standard, and have an 
interest in technology. We did not specify what it meant to 'have an interest in technology', thereby enabling our participants to self-select based on their own interests and curiosity about the project. Twenty-five older adults aged 70 and above were recruited.

3.1.1 Demographic Information. Demographic information on all 25 participants was collected via a questionnaire, either prior to the commencement of the workshops $(21 / 25)$ or between the first and second workshops (4/25). We also collected technology preference information for 21 of the 25 participants (four people did not fill out this questionnaire). All 25 participants were aged between 70 and 81 ( 11 female, 14 male). The demographic questionnaire asked them to nominate a pseudonym that would be used in reports or publications from the project. These pseudonyms are used in this paper.

3.1.2 Technology Experience. When asked about their experience with established computing technology, using a five point Likert scale ( $1=$ no expertise, $5=$ expert user), 16 participants rated their level of expertise on a smartphone/tablet as 3 and above. Expertise on a desktop computer was similar, with 17 participants rating their level of expertise as 3 or above. While this suggests that the participants had a high degree of self-confidence using computing technology, there was considerable heterogeneity within the group. For example, two respondents revealed they did not have access to a computer at all, and between four and five responses to each of the smartphone, tablet, and desktop questions indicated their expertise to be either 1 (no experience) or 2 .

Research conducted by the Australian Communication and Media Authority (ACMA) examining internet and device usage by older adults aged 65 and over has concluded this cohort is increasingly "embracing the digital life" [4]. For example, tablet usage by older adults now exceeds that of the adult population ( $18 \%$ as opposed to $16 \%$ ), and $68 \%$ of older Australians use a desktop or tablet computer to access the internet [4]. The report found these figures were similar to comparable studies conducted in both the United States of America and the United Kingdom [4]. These findings suggest that the level of digital literacy exhibited by our sample was broadly representative.

However, results from the questionnaire demonstrated a complete lack of familiarity with VR and avatars in the participant group. Participants were asked to rate their level of experience with VR technologies, virtual worlds and avatars using the same Likert scale approach. None of the participants had used a VR device of any type, and none had ever spent time in either a 2D or 3D virtual world. When asked about their experience of creating an avatar of any kind, no participants indicated ever having created an avatar previously. This led us to categorise the participants as 'curious novices' in relation to VR. Many had a general affinity with technology that they could build on, but none had direct experience of the technologies that would be used in the study.

\subsection{Workshop 1: Avatar Design}

Our first workshop invited older adults to create a humanoid avatar that they would later use in a social VR environment. The process of avatar creation and customisation is a common first step in many online virtual environments [44], particularly those involving social interaction [e.g. $12,22,23]$. Affording our participants the opportunity to design their own avatars was consistent with our methodological approach that aimed to give the older adults a voice in shaping the research program. It also supported our participatory goals by giving them the freedom to explore avatar designs that responded to their needs or curiosities, rather than design based on direction from the researchers. The only instruction given at the beginning of the avatar design workshop was that their avatars would serve as their individual self-representations in subsequent workshops that would seek their insights about social VR. Scheduling a workshop that required a fairly high degree of technical skill so early in the research program involved some risk. However, we felt this was outweighed by the benefit of giving the participants the ability to explore avatar design

Proc. ACM Hum.-Comput. Interact., Vol. 3, No. CSCW, Article 149. Publication date: November 2019. 


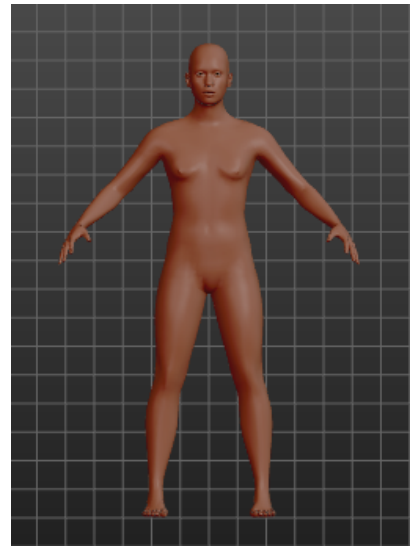

Fig. 1. Image of the stock humanoid body used as the base avatar in Workshop 1.

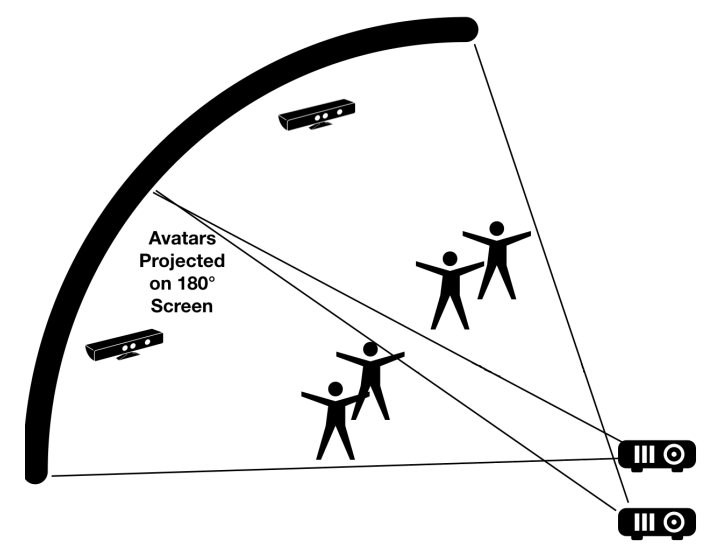

Fig. 2. Schematic layout of projected avatar prototype used in Workshop 2.

for themselves rather than selecting from pre-designed avatar types, a technique that had been employed in previous avatar focused work with older adults [17, 65].

Twenty-three of the 25 participants recruited for the project took part in the avatar design workshop, comprising a single 90-minute avatar design session. Participants were provided with individual computers that were pre-loaded with MakeHuman, an open source avatar creation program that allows users to adjust avatar characteristics using graphical sliders ${ }^{1}$. One member of the research team introduced the toolbars, tabs and sliders that applied to each 'section' of the avatar (e.g. body shape, clothes, facial features, hair etc.). Additional members of the research team observed the workshop and discussed the avatar designs with participants as it progressed. These team members were available to assist when participants asked for help. Each participant began with a humanoid avatar (see Figure 1) that they were free to customise from then on.

At the end of the workshop, the participants completed an online questionnaire that asked specific questions about their design motivations and the characteristics of their avatars that were most important to them. In this article we focus on participants' statements about their design motivations, comments made about their completed avatars, and reflections on the design process Findings related to the analysis of each avatar design and how the designs relate to the wider literature examining the use of avatars by older adults are discussed in [14].

\subsection{Workshop 2: Projected Avatar Prototype}

The second workshop took place eight weeks after the avatar design workshop. This gap was necessary to allow time for the development of the two social VR prototypes that would comprise workshops 2 and 3 described below. During the planning and reflection that took place after the avatar design workshop, it was clear that the participants were intrigued by the extent to which their avatars would have a sense of realism when they were under their control in a virtual environment. Based on this knowledge, we developed a prototype social virtual world that would facilitate participants coming face-to-face with their embodied avatars by standing in front of a large projection screen. This would allow them to gain a fuller appreciation of the way in which embodied avatars react to body movement in a virtual environment, a task that is much more difficult in VR environments that adopt a first-person perspective.

$\overline{{ }^{1} \text { http://makehumancommunity.org }}$ 


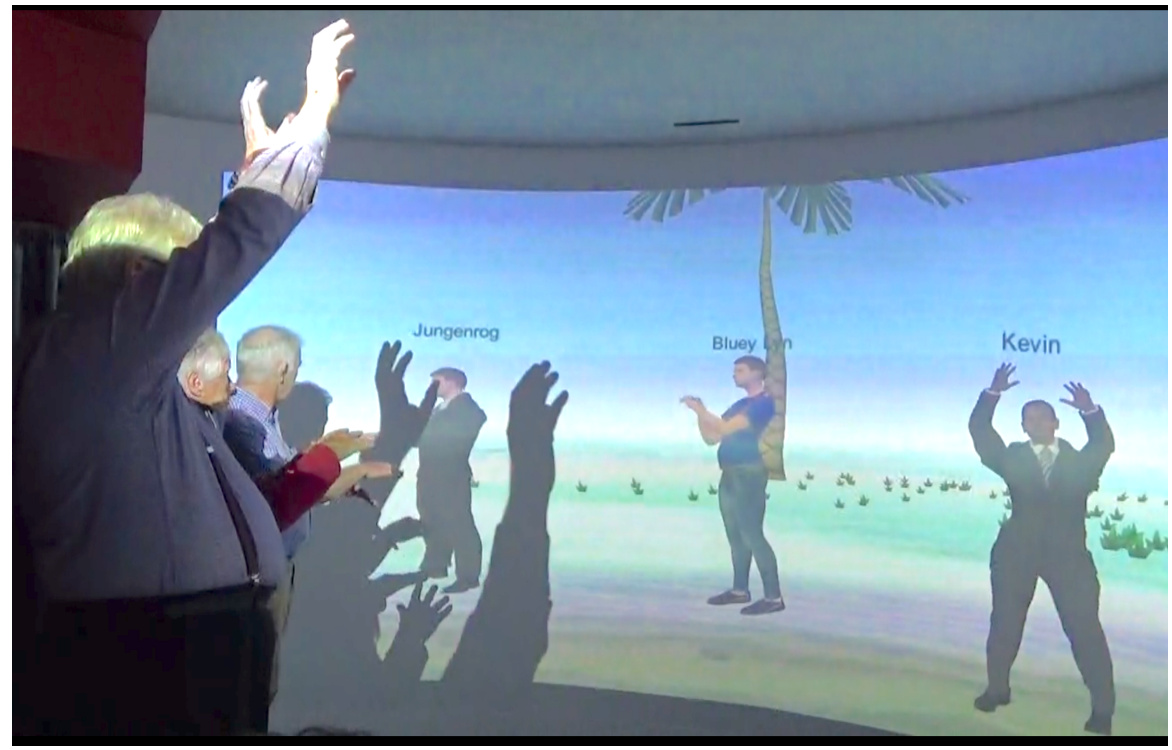

Fig. 3. Image taken showing some of the projected avatars in use during workshop 2.

Given the participants' lack of knowledge about virtual reality, workshop 2 also provided a opportunity to gradually introduce the participants to the idea of interacting in a simple virtual environment without the added complication of a HMD. The prototype consisted of groups of life-sized avatars, co-located in a projected virtual world and displayed simultaneously in two adjacent rooms fitted with 180-degree projection screens in a semi-circular shape. Figure 2 shows the setup used in each room, and Figure 3 shows an image of three participants using the prototype. The system allowed participants to communicate with other embodied avatars in a high-fidelity virtual world, and see how the embodied avatars moved using natural gestures. VoIP was used to create an audio link between the rooms to facilitate communication. Two Microsoft Kinect 3D cameras in each room were used to track participants' movements and map them to their avatars. The avatars from both rooms were then simultaneously projected onto the 180-degree semi-circular screen in front of each group.

Twenty-one participants attended the second workshop (four were either on vacation or caring for grandchildren). They were divided into groups of seven to make use of the prototype, with three individuals in the first room and four in the second. A facilitator was present in one of the rooms and was also embodied as an avatar to introduce participants to the features of the virtual environment and encourage social interaction. Sessions began with participants standing in front of the screen and looking at their avatars. During this time, the facilitator asked each participant-embodied as their avatar on the screen-to introduce themselves to the rest of the group and highlight their avatar via a 'signature' movement, e.g. a hand wave or dance move. This enabled each participant to understand and explore how their avatar would respond to their own body movements. Once all the avatars had been introduced, the group undertook a series of movements and unscripted conversations, allowing participants to provide spontaneous feedback on the technical capabilities of VR and the experience of being embodied as an avatar. After using the prototype for approximately 30 minutes, the participants took part in focus groups to capture 


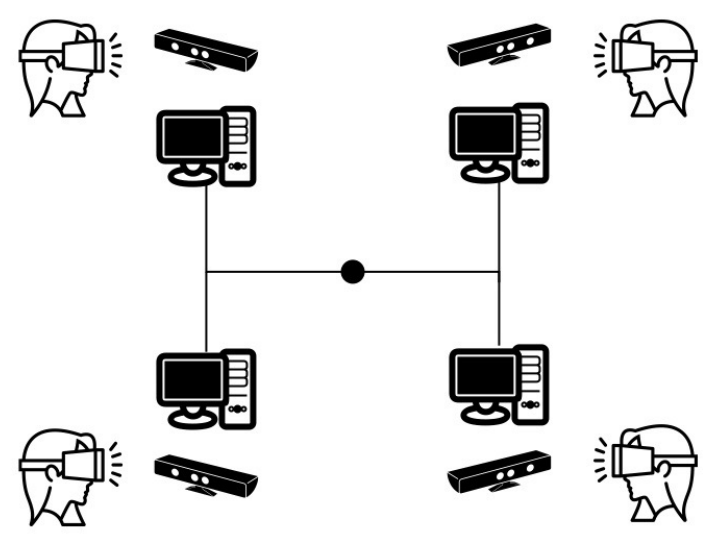

Fig. 4. Schematic of immersive avatar prototype used in workshop 3.

their reactions to the experience. A list of focus group questions that were used to initiate discussion after workshop two is included in the supplementary material that accompanies this paper.

\subsection{Workshop 3: Immersive Avatar Prototype}

The third and final workshop took place four weeks after the second workshop. In reflecting on the results from the second workshop, it was clear that participants had an improved understanding of how an embodied avatar would appear and move in a virtual world and an appreciation of the translation of embodied avatars into a virtual environment. We therefore felt confident about moving to a fully immersive social VR environment for the final workshop. This gave the participants the chance to explore a prototype social VR environment that used HMD technology to enable a fully immersive first-person perspective, as is common in commercial technologies such as the Oculus Rift and HTC Vive.

Figure 4 provides a schematic representation of the immersive prototype setup used in the workshop. Participants were seated in individual rooms equipped with HMDs that were connected to a computer running the virtual environment inside the Unity game engine. Each room also had a Microsoft Kinect 3D camera to track body movements, which were then mapped to the participants' avatars within the VR environment. Both HTC Vive and Oculus HMD were used in the prototype.

The virtual environment depicted in the prototype consisted of an island where the participants' avatars were seated around a reflective pond that allowed them to look down and see themselves (see Figure 5). Audio headsets enabled conversation between the participants via a 3D positional audio Unity plugin.

Twenty-two participants attended workshop three (one participant had withdrawn from the project and two were unavailable), and up to four participants took part in each session. A facilitator was also present in the social VR prototype to answer questions and introduce discussion topics. Each participant spent approximately 20 minutes using the prototype, during which time they looked through the eyes of their embodied avatars using the HMD, took part in group discussions about the features of the virtual environment and their colleagues' avatars, and experimented with various avatar gestures. Immediately following their time using the prototype, the participants took part in focus groups to discuss their reflections on the experience. 


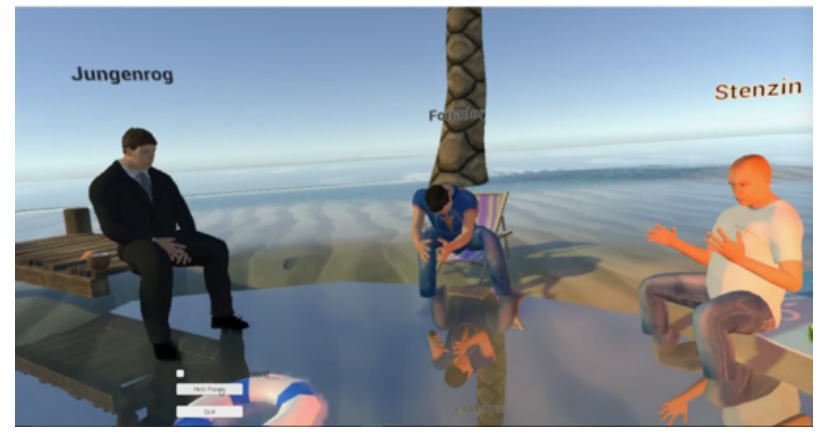

Fig. 5. Screenshot of immersive avatar prototype used in workshop 3.

\subsection{Data Collection and Analysis}

Our study employed multiple sources of data and methods of data collection. These included questionnaires, researcher notes, and focus groups. All data collected were transcribed (when necessary) and imported into QSR International's NVivo qualitative analysis software for coding and analysis. We conducted thematic analysis using Braun and Clarke's six-stage analytic approach [10]. Independent parallel coding was adopted for the study [69]. Three members of the research team coded a selection of raw transcribed data. All codes were then discussed in a review meeting to ensure a level of consistency between the codes [38]. In addition, a copy of the document detailing each stage of the Braun and Clarke process (completed by the first author) was sent to the entire research team so that all could interrogate any stage of the thematic analysi

In order to build in a level of co-enquirer review, a particularly important process given the participatory goals of the project, an early draft of this paper was sent to the participants as a stakeholder check [69]. Participants were encouraged to comment on how the reported findings related to their personal experiences. Seven participants responded to the draft. Some respondents offered whimsical observations, such as Klaus, who commented that he was "very much relieved that I wasn't the only grizzling old bugger amongst the lot of us". None reported any concerns about the way the findings portrayed their experiences of the prototypes. The participants explicitly commented that our central themes matched with their experiences, providing evidence that our themes reflected their views on the workshops. Bernard succinctly summarised the responses when he commented that "The reported findings and examples seem to fit my experiences and memories", while Samuel commented that "In my view your paper gives a good representation of the VR research intention, process and findings". An added advantage of the stakeholder check process was that it provided an opportunity to directly disseminate results back to the participants who were involved in the study.

\section{FINDINGS}

We present our findings across three subsections. First we discuss older adults' reactions to the workshops at a general level to illustrate the degree to which they felt involved in the participatory research program. We then explore our participants' perspectives on social VR alongside the potential value of the technology for older adults, before discussing issues related to avatar design and fidelity that impacted their experience. These subsections highlight a range of factors that will need to be addressed if social VR is to be a valuable communication medium for older users. 
Table 1. Self-reported Avatar Motivations and Participant Comments

\begin{tabular}{|c|c|c|}
\hline Self-reported Avatar Motivations & \# of Responses & Example comments \\
\hline \multirow[t]{5}{*}{ 'Realism' and personal likeness } & 9 & "A realistic figure" (Carl) \\
\hline & & "A somewhat idealised version of how I would like to be now" (Herbert) \\
\hline & & "Like Myself" (Holly) \\
\hline & & "A version of myself in my 20s" (Samuel) \\
\hline & & "Realism" (Ern) \\
\hline \multirow[t]{3}{*}{ Relations } & 3 & "My grandson closely resembles my avatar" (Thomas) \\
\hline & & "One of my granddaughters" (Mandy) \\
\hline & & "My avatar is Asian, [as is] my niece" (Tina) \\
\hline \multirow[t]{2}{*}{ Alter-egos } & 3 & "A companion for travelling, both in reality and VR" (Bernard) \\
\hline & & "Someone very strong and threatening" (Herb) \\
\hline \multirow[t]{3}{*}{ Playful/Testing the software } & 5 & "As it was the first avatar I created, I was more interested in process" (Nik) \\
\hline & & "Getting familiarity with the software" (Klaus) \\
\hline & & "Just having fun playing with the software" (Amy) \\
\hline
\end{tabular}

\subsection{Participant Reactions to the Workshops}

4.1.1 Avatar Design Process and Motivations. At the conclusion of the first workshop, participants were asked to complete an online questionnaire containing seven short response questions that sought to understand their motivations while going through the avatar design process, their reflections on the workshop experience, and any thoughts on the limitations presented by the design software being used (refer to supplementary material to view the questions asked in the questionnaire). When participants were asked "Do you have any comments regarding your avatar, or the workshop, that you would like to share?", many participants gave positive feedback about the process and their ability to make individual choices. Herbert compared the avatar creation process to his hobby of sculpting wood, suggesting he felt in control of the process and was able to shape his avatar in personally satisfying ways. Violet commented that the workshop had been "a most enjoyable initiation into avatars", while Harry appreciated that "the workshop went at a pace that everyone could keep up with". Finally, Bernard believed the workshop "was enjoyable and fulfilling because while we were pointed in various directions, there was no attempt to make us do [a] particular thing. The choices were ours". These findings suggest that our participatory approach was valued as it allowed the participants to make their own choices within the context of the research program. Furthermore, responses indicate that participants felt comfortable using a system that was potentially challenging, despite its novelty and considerable latitude for enabling avatar customisation.

In the online questionnaire conducted at the conclusion of the avatar design workshop, the participants were also asked about their design motivations. Twenty participants responded to the survey. Participants' design motivations fell into four categories that are listed in Table 1 along with comments they made about each of these categories. The most commonly stated motivation was to create 'realistic' avatars. These avatars represented attempts to visually mimic the participants' conceptions of the way they looked in real life. Some were motivated to create avatars that resembled relatives-most commonly grandchildren-or some sort of alter-ego. Finally, five participants were motivated by a more playful approach. When describing the motivations behind these playful avatar designs, participants in this group tended not to relate the avatars directly to themselves, but rather used the design session as an opportunity to test the possibilities afforded by the software. A more detailed account of the avatars designed by the participants and a full analysis of what these designs suggest about how older adults wish to be represented in avatar form is reported in [14]. 
4.1.2 General Reflections on the Social VR Experience. Workshops 2 and 3 represented participants' first exposure to immersive social VR technologies. At the conclusion of the sessions, many participants expressed a sense of amazement about the sheer technical accomplishment of being able to control their avatars through natural gestures. This was perhaps best illustrated by Len, who began by saying "If I told my father about [the experience] he'd think I came from Mars, and yet we're starting to say this is real... it's intriguing in our age group where you can actually say, there's something on the screen that is responding to my actions". Klaus was fascinated by the technical aspects of the system, commenting that "...it's quite amazing the amount of data that the camera must pick up to... accurately [track] the movements of the avatar". Kim commented on how enjoyable she found the experience: "...this is... it's just intriguing the whole thing... it's just great fun and very enjoyable".

Reactions to the immersive avatar prototype (workshop 3) centred around the nature of the first person view through the HMD and the fact that it 'transported' the participant to another world. For Samuel, this experience helped to crystallise the potential of social VR for older adults: "As you get older, you're less mobile and you can see... you can bring people together in [social VR] ... you'd [feel] 'I'm not going to get on the bus... but let's get together in [social VR]'." Herb's comments revealed him grappling with the implications of the immersive experience: "You almost feel like it was reality for a while, that you immersed yourself in what you were doing and you became, not necessarily a person... but [the other avatars] were the people you were talking to". These responses affirmed that the group was indeed curious and excited about technology, and more specifically, provided evidence that the workshops provoked curiosity about the potential of social VR as a tool that might benefit older adults. This aspect of the study is explored in more detail below.

4.1.3 Comments About Usability. Despite using the prototype for around 20 minutes in each workshop session, no participants reported any dizziness or symptoms of simulator sickness $[32,33]$. However, there were several comments related to issues encountered while using the HMD. Some participants were unsure as to whether they should leave their glasses on or off when using the headset. Sally, who removed her glasses, regretted the decision, commenting later that everything appeared "soft", suggesting she was unable to satisfactorily find a HMD position that would allow her to bring the virtual world clearly in focus. This highlights a core problem related to assisting users in VR as although there was a researcher present during the session to assist the participants, they have no way of seeing focal issues directly and must simply show the participant ways to adjust the focus of the HMD and trust they will find a suitable setting. Only Amy reported discomfort from wearing the HMD for an extended period, commenting that "I found the headset was mildly distracting. It's a bit uncomfortable". Despite several participants having issues related to hearing loss, sound quality in the prototype was rated as being good, although several participants commented that they were not aware of the direction of the speaker's voice, despite the prototype employing spatial audio software.

\subsection{Insights into the Role Social VR Can Play in Older Adults Lives}

One of the goals of our study was to engage the participants in a conversation about how, based on their experience, social VR might contribute positively to the lives of older adults. Bernard felt the consistency of an avatar's appearance and the ability to model a consistent virtual environment could help those with dementia to remember names and places as "they would always look the same". Cleo - who lived in an aged care facility - disagreed with this assessment, stating that some of the people she lived with "have their own reality" and were happy. She felt that the social VR environment could be distressing for these people. Samuel, on the other hand, believed that avatars could provide a means for health care professionals to gain a better understanding of what it was 
like to be an older person and this experience might lead to them "treat[ing] their patients a lot differently". Samuel's comments demonstrate that he recognised the potential of social VR as a tool for perspective taking [55], but with a specific focus on the way it might be used to allow others to better understand the lived experiences of older adults.

The immersive avatar prototype used in workshop 2 inspired additional comments about how social VR might respond to the needs of older adults. Several participants wondered how an avatar might allow for alternative methods of self-representation. Herb suggested that avatars offered the potential to improve feelings of self-worth, noting that "if you had an [avatar] of somebody that you looked up to and thought was wonderful and you talked [through] them... you might be able to do lots of things because your self-esteem would go up". Samuel suggested that "there might be times when you'd much rather send your idealised (avatar) self out... Particularly if you become disabled". He related this to the story of an older colleague who stopped visiting work friends because she had a "droopy face" after she suffered a stroke. Researchers have noted that some older adults can become socially isolated due to self-consciousness about the impacts of ageing or a specific age-related disability [20]. Herb and Samuel's comments suggest a potential role for social VR and avatars in providing alternative avenues for older adults to maintain social connections while also exercising control over their appearance.

\subsection{Embodied Avatars as Social Agents}

While participants generally reported enjoying the experience of creating their avatars and embodying them in the social VR workshops, our analysis revealed a number of concerns. One such concern brought forth by the workshops related to the visual appearance and expressive qualities of the avatars within the social VR prototypes. Another concern related to the ability for older adults to accurately control their avatar in the prototypes, alongside the impact that body tracking errors can have in undermining the participants' control over the ageing body and the way in which this lack of control might conjure negative stereotypes about ageing. These findings revealed our participants keen interest in creating embodied avatars that they perceived to be realistic, and which portrayed their behaviour in an authentic and meaningful fashion that demonstrates they are in control of their avatars and, by extension, their own bodies.

4.3.1 Searching for Realism. Even though participants were yet to 'use' their avatars, comments made during workshop 1 and in the post-workshop survey highlighted an immediate desire for the participants' avatars to be more expressive.

At the conclusion of the avatar design workshop, participants were asked whether there was any aspect of their avatar that they would most like to change. One participant, Tina, replied that her avatar was "too android in appearance". Bernard similarly described his avatar as not looking "happy" and referred to it as having "a sour look". Ern described his avatar as having an "unsmiling mouth and eyes", which he hoped might be able to be "achieved with animation". Ern's comment in particular suggests that some of this dissatisfaction might simply have been a result of not having the ability to animate the avatar during the design session. However, data from later workshops demonstrated this was a consistent theme running through the study.

Just as in the avatar design workshops, the 'realism' of the participants' avatars and their ability to reflect a 'real personality' were major themes relating to the projected avatar prototype used in workshop 2. Only Herb, who designed an alter-ego avatar character, expressed contentment with his avatar, stating "I've always been such a timid little person. To have [an avatar] who is bold and strong, I can really live something totally different. My other person could come out a bit further". For the majority who created avatars designed to more closely reflect their own appearance, the prototype experience was soured by the feeling that their avatars lacked "vitality". 
This often related to the embodied avatars' lack of facial expression. Carl commented that "because [his avatar] doesn't have any facial expressions it's very hard to work a personality out". Len also drew attention to the lack of facial expression: "Well there were a fair few limitations, the fact that there were no facial expressions." A common complaint from several participants was that the lack of "personality' rendered the avatars as mere "puppet(s)" (Gordon) or "marionette(s)" (Tina). This tendency for thinking of avatars as puppets or robots has been observed in similar studies of older adults' reactions to avatars in social contexts [65]. In fact, when asked for the one feature they would change if they had a magic wand, almost all the participants called for options to give their avatars more expressiveness and realism. The following exchange is reflective of these comments:

Violet: Without facial expression, it makes a big difference, because that's usually what is communicating.

Samuel: Yeah, I thought that exactly...Emotion is very much tied up in personality...[the avatars] all seemed like zombie[s] [they] lacked vitality, literally!

Facilitator: How do you think we could give them more vitality?

Herb: Facial expressions.

As occurred in workshop 2, many comments from the participants during the immersive avatar prototype (workshop 3) highlighted the importance of 'realism' in promoting genuine social interactions. While comments from some participants indicate they felt fully immersed within the prototype that employed HMDs (see section 4.1.2), the perceived lack of realism in the avatars prevented others from having this experience. Amy's comments were illustrative of this. After removing her HMD, she commented that the experience was "Very artificial. Unbelievable... there were no clues in the movements of the avatars to say 'look over there because that's who's talking'.. It was like talking to a group of dolls. Mechanical dolls". Holly's comments echoed these concerns: "to get any real benefit out of [social VR], you'd have to believe that [the avatar represented] that person. They would have to show some sort of emotion... there wasn't the interaction". Others were more forgiving of the technical limitations of the prototype, but still emphasised the need for improved expressiveness in the avatars. Bernard commented that "[as] technology improves, those things are going to change. The big thing is, how are you going to get to... see facial expressions [when] you want to talk to someone?". These findings reinforce prior work that has highlighted older adults' keen interest in online social interactions that give them a rich sense of communicating with another person $[11,31]$. The perceived social value of the VR prototypes was greatly eroded in cases where avatars were considered to be mechanical, sallow, or un-lifelike.

4.3.2 Reacting to Social VR Tracking Errors. The Kinect 3D cameras that tracked body movements in the prototypes at times exhibited tracking errors and jitters that caused the participants' avatar bodies to twitch, contort, and shake. Interestingly, participants frequently made sense of these involuntary movements in terms of what they might mean about the 'personality' of their avatars. For some, like Mandy, the randomness of the tracking errors gave the avatar a playful characteristic and allowed it to move in ways that she no longer could:

My avatar could do all sorts of things... without being told, she just did the splits and jumps...[the avatar] was very independent, she did what she wanted to do... it was cute.

For Harry and Holly, however, the tracking errors led to a stiffness and jerkiness that they found disconcerting. Harry commented that the avatars weren't performing an "accurate representation of my movement".

Participants' reactions to these tracking errors were even more pronounced during their use of the immersive avatar prototype (workshop 3). Some participants commented on the impact that tracking errors and jitters had on their sense of control over their virtual bodies. Thomas 
expressed frustration about not being able to accurately control his hand movements as they were key non-verbal communication tools. Carl responded by saying he felt he "had developed Parkinson's disease and my hands were shaking, but involuntarily". Frustration at the inability to control fine hand movements was a common theme in the data and led to more comments about the robotic nature of the avatars. As Bernard described, "[it's] robotic Dr Who sort of stuff isn't it.. I mean you could do [any movement] with your fingers and it didn't show up [on your avatar]."

These findings raise issues about the current state of body tracking technology in VR, and about older adults' specific anxieties with respect to the ageing body. With regard to body tracking technology, while tracking errors of differing severity impact on the usability of all commercially available VR systems, our findings highlight how damaging tracking errors can be in social VR systems where users are looking to convey social meaning via non-verbal cues. Our findings illustrate the particular sensitivity our participants had to involuntary movements caused by tracking errors. Older adults are particularly sensitive to social stereotypes that render the ageing body as being an object of disgust that makes them "liable to sanctions, both physical and symbolic" [70, p.128]. Thus, in social VR, tracking errors are not simply seen as technological 'glitches' by older users, but may mark them as objects to be avoided or ostracised.

\section{DISCUSSION}

The aim of this work was to investigate older adults' preferences regarding immersive social VR technologies. We seek to use these findings to inform the future development of social VR as a communication tool. By exposing a group of older adults to the process of avatar creation, alongside an experience of interacting with others via two social VR prototypes, our study provides several key insights that can support the design of social VR as a communication medium for use by this population. The first insight is the significance of behavioural anthropomorphism in social VR and its importance in addressing older adults' desire for expressive, human-like communication. The second insight is the importance of translational factors - how VR technology translates the movements of the ageing body into the virtual environment - as a key factor for supporting the quality of social interaction experienced by older adults in social VR. In the discussion below we develop these insights further before considering how social VR designs can support older adult communication. We then consider how older adults' views can inform future research priorities within this space.

\subsection{Behavioural Anthropomorphism as the Key Aspect of Older Adults' Embodied Avatars in Social VR}

One of the themes that permeated most strongly throughout the study was the importance that participants placed on the need for their embodied avatars to be able to move and act in more 'human' ways when interacting in a social VR environment. Just as the wooden toy Pinocchio in Carlo Collodi's children's novel dreamed of becoming a 'real boy', the participants sought to inject more personality and reality into their avatars. Many felt that the avatars used in the prototypes lacked these important characteristics, rendering their avatars more like 'marionettes', 'puppets', or 'mechanical dolls' than self-representations. The participants expressed these views via the use of terms such as: 'realism', 'vitality', 'personality', and by emphasising the need for facial expressions. Translating terms such as these into specific design considerations can be difficult as it is not always clear what a comment like 'realism' means in relation to an embodied avatar [53].

In their recent review of the terminology used to describe avatar characteristics, Nowak and Fox recommend researchers discuss avatars within the context of three "overlapping aspects that influence users' perceptions of the social potential of avatars" [53, p.36]. These aspects are: agency (is the avatar perceived as human?), anthropomorphism (does the avatar have human form or 
behaviour?), and realism (does the avatar have accurate form or behaviour). They distinguish anthropomorphism and reality by noting that an avatar of a tree may be highly "accurate and lifelike" and thus realistic; however, "other users may be less likely to attribute social potential to it-and [be] less likely to...communicate with it-because it is not anthropomorphic" [53, p.37].

Thus, while the participants used terms such as 'realism', 'vitality', and 'personality', we might better understand these as descriptions relating to anthropomorphism and specifically behavioural anthropomorphism, which describes "avatars speaking, moving, or acting in ways that may be expected of humans" [53, p.36]. Prior literature has established that older adults prefer technological systems that support complex identities and deeper, more personalised, social communications $[11,31]$. Our study highlights how these concerns and wishes play out in the specific context of social VR as a desire for behavioural anthropomorphism. Our findings, allied to the insights from prior literature, are enough to suggest that behavioural anthropomorphism is one of the foremost concerns of older adults with respect to social VR.

\subsection{Translating the Movement of the Ageing Body in Virtual Environments}

While behavioural anthropomorphism speaks to the need for embodied avatars to move and act in 'human' ways in order to support communication between older adults, our study also highlights the importance placed by older adults on having VR systems translate the movement of the ageing body in ways that avoid perpetuating negative ageing stereotypes. After participating in workshop 2, Samuel discussed a colleague who withdrew from contact with former workmates because of the physical impacts of a stroke. Samuel wondered if embodied avatars might allow older adults impacted by physical disabilities to reengage with social supports. Prior research has established that older adults with age-related disabilities can become socially isolated due to these factors [20]. This supports the view that embodied avatars might play a positive role in aiding older adult communication in social VR by providing mechanisms that allow older adults to adapt their embodied movements to counteract the impacts of age-related disabilities. However, our findings also highlight that poor translation of bodily movements by body tracking hardware can severely impact on older adults' experiences in social VR by implying movements that correspond to negative social stereotypes, and which may associate "negative bodily qualities of oldness" with personal failure [34, p.14]. A prime example from our study was Carl's concern that the jitter experienced by his avatar suggested that he had Parkinson's disease.

In one of the few prior studies examining the social interaction experiences of older adults in virtual environments, Siriaraya and Ang have proposed that there are two factors that "affect the quality of the social interaction experienced by older people in 3D virtual worlds" [65, p.104]. These are factors that are related to the characteristics of the users (User factors) and factors that are related to the design of the virtual environment (Platform factors) [65]. Our findings suggest that consideration of a third set of factors - what we might term Translational, or how the technology converts bodily movements from the real world into the virtual environment - is required in order to ensure that social VR hardware and software can accommodate the self-presentational concerns of older adults. For example, some older adults may desire 'smoothing' of avatar movements to reduce the signs of physical tremor, particularly if these adults internalise and feel subject to ageist stereotypes. Others may wish for their movements to be accurately recreated within the VR environment, either because their disabled identity is important to them or because they are able-bodied and want to be represented as such. This level of translational control will empower older adults themselves to choose the nature of their embodied avatar's movements, and thus how they wish to be represented within a social VR environment.

As a result of this study, we believe that behavioural anthropomorphism and addressing factors relating to the translation of the movements of the ageing body are key challenges facing those 
designing embodied avatars and social VR systems that support communication between older adult users. As the field of social VR research is still evolving, a key contribution of our study is to propose a set of design considerations that seek to begin addressing these challenges when designing social VR systems aimed at older adults. This will also help to situate social VR within the broader context of designing to support older adult communication, as discussed below.

\subsection{Designing to Support Older Adult Communication in Social VR}

The need for embodied avatars to support behavioural anthropomorphism, though indicative of a new form of computer mediated communication, nonetheless resonates with the broader literature examining older adults communication preferences. In particular, our findings emphasise the importance of "heavyweight" communication to older adults and their need to communicate in ways that leave them feeling "that real contact has been made with someone" $[41,1707]$. Our findings suggest that improved behavioural anthropomorphism is the gateway to heavyweight communication when using embodied avatars in social VR. Three key areas of design appear to be crucial.

5.3.1 Capturing or Inferring Facial Expressions. Our participants were unanimous in seeking improvements in capturing facial expression in order to help them to feel a sense of genuine connection with their own, and others', embodied avatars. While social VR systems may evolve in the future in ways that mean a HMD is not necessary, at present, the need to wear a HMD obscures much of the face. This presents an engineering challenge when trying to read or infer facial movement. Those designing to support behavioural anthropomorphism in social VR must therefore consider ways to translate facial movement onto embodied avatars while the user is wearing a HMD. Current approaches to this challenge vary from embedding sensors inside the HMD that measure surface tension of the face [39], to embedding photo-reflective sensors [68]. Until these challenges are met in commercially available systems, those designing social VR experiences for older adults should at least attempt to maximise embodied avatar facial expressiveness by:

- Employing lip syncing technology that uses audio signals to infer avatar mouth movements. For example, plugins, such as Oculus Lipsync, allow designers to use a set of phonetic mouth shapes (called 'visemes') to simulate natural mouth motion based on the incoming audio signal $^{2}$.

- Adopting software that can infer or simulate eye movement. Recent research suggests that avatars with simulated eye movements can result in "high quality of communication" in co-located social virtual reality, even when compared to real eye movements captured with eye-tracking software [62, p.531].

These design goals begin to incorporate a level of facial expression into embodied avatars, moving us closer to the level of behavioural anthropomorphism that is desired by older adults.

5.3.2 Accommodating Nonverbal Communication. While the prototypes employed in this study did track body movements, the participants, particularly in the immersive prototype (workshop 3), called for a finer level of tracking - particularly tracking of hand gestures. Psychological research has demonstrated the pivotal role that nonverbal cues such as hand gestures play in helping people judge rapport in new social circumstances [27].

The need to understand the importance of hand gestures represents a design consideration that has not been a factor in evaluations of other social communication technologies for older adults. It is an example of what Hope and colleagues refer to as the need for designers to consider the "rapidly changing communications landscape, where the technologies of today will be replaced

$\overline{{ }^{2} \text { https://developer.oculus.com/documentation/audiosdk/latest/concepts/audio-ovrlipsync-viseme-reference/ }}$ 
by new interaction paradigms of tomorrow" [31, p. 3910-3911]. Our findings foreshadow a new social communication landscape in which nonverbal gestures play an ever increasing role. As such, we suggest that those designing social VR experiences for older adults consider using VR hand controllers which, though adding another level of complexity with respect to interaction, allow older adults to use precise hand gestures to help convey meaning and build rapport in social virtual worlds. An added advantage of this recommendation is that it allows older adults to hold and manipulate virtual artefacts. Given the noted desire amongst older adults to share material artefacts [31], the use of hand controllers also opens up a new design space where scanned artefacts and interactive games can further promote heavyweight communication by allowing them to bring meaningful 'real world' objects into social VR to further enrich the social experience.

5.3.3 Designing for the Ageing Body. A final design consideration relates to the need to consider how older adults will interpret bodily movement in social VR. Our findings illustrate the distinct ways older adults interpreted the tracking errors that were a byproduct of the Kinect sensors. These ranged from Mandy's positive reaction to her avatar's propensity to "do the splits and jumps" through to Carl's concern that his avatar had Parkinson's disease.

While the majority of the participants' responses undoubtedly called for more accurate body tracking, the range of reactions to the tracking in the prototypes suggests to us that social VR affords a unique opportunity to consider how tracking might be used to allow older adults choice in how closely their embodied avatars mimic their movements. Thus, we believe that future social VR experiences should be designed in such a way that body tracking can be adaptive and that Translational factors - in addition to User and Platform factors [65] - are central to the development of social VR experiences aimed at older adults. This will allow systems to prevent the types of tracking errors that might conjure negative stereotypes related to the ageing body (such as Carl's), while affording a level of control that could allow those suffering from physical disabilities (such as those related to Parkinson's disease or stroke) to present themselves as they wish in social VR.

\subsection{Older Adults' Views Informing Future Research Priorities}

In their critical analysis of 30 years of ageing research in the HCI community, Vines et al. call on researchers to "engage with how older people themselves might actively direct, shape, and contribute to HCI research" [74, p.21]. We believe our engagement with the participants demonstrates a practical method that can move HCI research toward this goal. By asking the participants directly about their thoughts on the potential of the technology after engaging with the prototypes, they provided views that demonstrate how participatory engagement of this type can be used to help inform future research in a range of areas involving the use of social VR.

5.4.1 VR, Ethics and Dementia Care. Bernard and Cleo expressed a range of views about the benefits and challenges of using social VR with people who have dementia. The potential to use VR to support people living with dementia has been a topic of great interest in the area of gerontology $[43,49]$, and is emerging as a topic of interest in HCI [30]. As such, it is valuable to hear older adults' views on how VR might best be applied. We find Cleo's comments in particular very revealing. As an insider, living in a residential aged care facility with many people who are living with dementia, her feeling that VR might negatively impact on people who are already living in "their own reality", highlights an important ethical consideration for researchers working with this vulnerable population. Based on this learning, we believe that while our participants enjoyed their social VR experience, we should not view it as universally appropriate. We need more research to understand, in a more nuanced way, how and when social VR is appropriate, especially when VR is used in sensitive settings such as aged care $[59,76]$. 
5.4.2 Using VR to Improve Self-Perception and Wellbeing. Herb's views about the potential impact of embodied avatars on self-worth resonates with research that has demonstrated the profound impact that embodying an avatar can have on self-perception [55]. Samuel's comments about the contribution embodied avatars could make in encouraging older adults to reengage with social support provide evidence of the important role that social VR could play in improving wellbeing Research is increasingly demonstrating the positive role that embodied avatars can play in reducing anxiety [5] and establishing trust [37]. Our participants' comments provide first-hand accounts from older adults demonstrating their belief that such interventions could prove valuable for them and their peers.

5.4.3 Using VR to Better Understand the Ageing Experience. Finally, Samuel's comment that health professionals could benefit from embodying an older adult avatar also suggests a new focus for researchers seeking to understand the impact of VR on empathy [1,28]. Given that healthcare professionals' attitudes toward older adults have been shown to improve with increased knowledge of age-related issues [42], Samuel's insight provides an avenue by which VR training might improve how healthcare professionals respond to older adults.

\subsection{Limitations}

While our study has generated a rich set of data by which we can better understand older adults' use of embodied avatars in social VR, there are a number of limitations of the present study that can be addressed by future research. One such limitation concerned the design of the virtual worlds used in the prototypes. While the participants knew they would be embodying their avatars in prototypes before the avatar design workshop, they did not know the characteristics of the virtual worlds as they were yet to be developed. Based on these limitations, we are conducting extended participatory design work with the participants aimed at developing a social VR application that facilitates the types of social experiences they value. A second limitation relates to our conscious decision to use Kinect 3D cameras to track body movements in both prototypes. This approach was taken in order to improve the usability of the prototypes by allowing the participants to control their embodied avatars' movements via natural body gestures, rather than more complex combinations of technologies such as hand controllers and room-scale tracking. However, this decision to make the system easy-to-use came at the expense of acquiring more detailed insights about the usability of VR technologies by older adults. Such insights are likely to be valuable given the prevalence of hand controllers in commercial VR systems, and the known impacts that age-related illnesses, such as rheumatoid arthritis, can have on some older adults' use of hand-held devices [77]. To address this limitation, future work should study the usability issues raised by more complex tracking and control technologies.

\section{CONCLUSION}

In this paper, we present the findings of a study that sought to understand how social VR might be used as a communication tool that could contribute to the wellbeing of older adults. Twentyfive older adults aged over 70 took part in the study. We adopted a participatory action research approach and conducted three workshops that sought to give our participants the opportunity to design their own personalised embodied avatars and then use these in two social VR prototypes. Our findings highlight older adults' design motivations when creating embodied avatars for social VR; their views on social VR as a communication tool; and their thoughts about how social VR can play a beneficial role in the lives of older adults. Findings emphasise the critical importance placed by the participants on behavioural anthropomorphism, which encompasses their avatars' ability to speak, move, and act in a human-like manner in social VR. Our findings also highlight 
the critical importance our participants placed on have a VR system translate their movements in such a way as to avoid perpetuating ageing stereotypes. Based on this finding, we propose a set of design considerations for those exploring social VR as a communication medium for older adults. By examining the older adults' reactions and thoughts after interacting in the two social VR prototypes, we have also demonstrated how their views can inform a future HCI research agenda examining the use of social VR by older adults.

\section{ACKNOWLEDGMENTS}

This work is supported by an ARC Discovery grant (Project ID DP160101368). The first author would like to thank Zaher Joukhadar and Martin Reinoso for their technical support during the workshops. We also thank the anonymous reviewers for suggestions that improved this paper. Finally, we would like to thank all the participants who took part in the study for their time and contributions to the project.

\section{REFERENCES}

[1] Sun Joo Ahn, Amanda Minh Tran Le, and Jeremy Bailenson. 2013. The effect of embodied experiences on self-other merging, attitude, and helping behavior. Media Psychology 16, 1 (2013), 7-38.

[2] Aloha Hufana Ambe, Margot Brereton, Alessandro Soro, Laurie Buys, and Paul Roe. 2019. The Adventures of Older Authors: Exploring Futures Through Co-Design Fictions. In Proceedings of the 2019 CHI Conference on Human Factors in Computing Systems (CHI '19). ACM, New York, NY, USA, Article 358, 16 pages. https://doi.org/10.1145/3290605.3300588

[3] Aloha Hufana Ambe, Margot Brereton, Alessandro Soro, Min Zhen Chai, Laurie Buys, and Paul Roe. 2019. Older People Inventing Their Personal Internet of Things with the IoT Un-Kit Experience. In Proceedings of the 2019 CHI Conference on Human Factors in Computing Systems (CHI '19). ACM, New York, NY, USA, Article 322, 15 pages. https://doi.org/10.1145/3290605.3300552

[4] Australian Communications and Media Authority (ACMA). 2016. Digital lives of older Australians. Technical Report. 1-13 pages.

[5] Laura Aymerich-Franch, René F. Kizilcec, and Jeremy N. Bailenson. 2014. The relationship between virtual self similarity and social anxiety. Frontiers in Human Neuroscience 8 (2014), 944. https://doi.org/10.3389/fnhum.2014.00944

[6] Steven Baker, Jeni Warburton, Jenny Waycott, Frances Batchelor, Thuong Hoang, Briony Dow, Elizabeth Ozanne, and Frank Vetere. 2018. Combatting social isolation and increasing social participation of older adults through the use of technology: A systematic review of existing evidence. Australasian fournal on Ageing 37, 3 (Sept. 2018), 184-193.

[7] Steven Baker, Jenny Waycott, Sonja Pedell, Thuong Hoang, and Elizabeth Ozanne. 2016. Older People and Social Participation: From Touch-Screens to Virtual Realities. In Proceedings of the International Symposium on Interactive Technology and Ageing Populations (ITAP '16). ACM, New York, NY, USA, 34-43. https://doi.org/10.1145/2996267. 2996271

[8] Michel Benoit, Rachid Guerchouche, Pierre David Petit, Emmanuelle Chapoulie, Valeria Manera, Gaurav Chaurasia, George Drettakis, and Philippe Robert. 2015. Is it possible to use highly realistic virtual reality in the elderly? A feasibility study with image-based rendering. Neuropsychiatric Disease and Treatment 11 (2015), 557-563.

[9] Katherine Bessière, A Fleming Seay, and Sara Kiesler. 2007. The ideal elf: Identity exploration in World of Warcraft. Cyberpsychology \& Behavior 10, 4 (2007), 530-535.

[10] Virginia Braun and Victoria Clarke. 2006. Using thematic analysis in psychology. Qualitative Research in Psychology 3 , 2 (Jan. 2006), 77-101.

[11] Robin Brewer and Anne Marie Piper. 2016. "Tell It Like It Really Is": A Case of Online Content Creation and Sharing Among Older Adult Bloggers. In Proceedings of the 2016 CHI Conference on Human Factors in Computing Systems (CHI '16). ACM, New York, NY, USA, 5529-5542. https://doi.org/10.1145/2858036.2858379

[12] Barry Brown and Marek Bell. 2004. CSCW at Play: 'There' As a Collaborative Virtual Environment. In Proceedings of the 2004 ACM Conference on Computer Supported Cooperative Work (CSCW '04). ACM, New York, NY, USA, 350-359. https://doi.org/10.1145/1031607.1031666

[13] Kelly E. Caine. 2009. Supporting Privacy by Preventing Misclosure. In CHI '09 Extended Abstracts on Human Factors in Computing Systems (CHI EA '09). ACM, New York, NY, USA, 3145-3148. https://doi.org/10.1145/1520340.1520448

[14] Romina Carrasco, Steven Baker, Jenny Waycott, and Frank Vetere. 2017. Negotiating Stereotypes of Older Adults Through Avatars. In Proceedings of the 29th Australian Conference on Computer-Human Interaction (OZCHI '17). ACM, New York, NY, USA, 218-227. https://doi.org/10.1145/3152771.3152795 
[15] Romina Carrasco, Jenny Waycott, Steven Baker, and Frank Vetere. 2018. Designing the Lost Self: Older Adults' Self-representations in Online Games. In Proceedings of the 2018 Designing Interactive Systems Conference (DIS '18). ACM, New York, NY, USA, 441-452. https://doi.org/10.1145/3196709.3196773

[16] Ying-Yu Chao, Yvonne K. Scherer, and Carolyn A. Montgomery. 2015. Effects of using Nintendo Wii ${ }^{\mathrm{TM}}$ exergames in older adults: a review of the literature. fournal of Aging and Health 27, 3 (April 2015), 379-402.

[17] Wei Lun Cheong, Younbo Jung, and Yin-Leng Theng. 2011. Avatar: A Virtual Face for the Elderly. In Proceedings of the 10th International Conference on Virtual Reality Continuum and Its Applications in Industry (VRCAI '11). ACM, New York, NY, USA, 491-498. https://doi.org/10.1145/2087756.2087850

[18] José Coelho and Carlos Duarte. 2016. A literature survey on older adults' use of social network services and social applications. Computers in Human Behavior 58 (May 2016), 187-205.

[19] Nicole Cook and Sandra L. Winkler. 2016. Acceptance, usability and health applications of virtual worlds by older adults: a feasibility study. FMIR Research Protocols 5, 2 (2016), e81.

[20] Erin York Cornwell and Linda J. Waite. 2009. Social disconnectedness, perceived isolation, and health among older adults. Journal of Health and Social Behavior 50, 1 (March 2009), 31-48.

[21] Elizabeth V. Cyarto, Frances Batchelor, Steven Baker, and Briony Dow. 2016. Active Ageing with Avatars: A Virtual Exercise Class for Older Adults. In Proceedings of the 28th Australian Conference on Computer-Human Interaction (OzCHI '16). ACM, New York, NY, USA, 302-309. https://doi.org/10.1145/3010915.3010944

[22] Nicolas Ducheneaut and Robert J. Moore. 2004. The Social Side of Gaming: A Study of Interaction Patterns in a Massively Multiplayer Online Game. In Proceedings of the 2004 ACM Conference on Computer Supported Cooperative Work (CSCW' 04). ACM, New York, NY, USA, 360-369. https://doi.org/10.1145/1031607.1031667

[23] Nicolas Ducheneaut, Ming-Hui Wen, Nicholas Yee, and Greg Wadley. 2009. Body and Mind: A Study of Avatar Personalization in Three Virtual Worlds. In Proceedings of the SIGCHI Conference on Human Factors in Computing Systems (CHI '09). ACM, New York, NY, USA, 1151-1160. https://doi.org/10.1145/1518701.1518877

[24] Patricia M. Eng, Eric B. Rimm, Garrett Fitzmaurice, and Ichiro Kawachi. 2002. Social ties and change in social ties in relation to subsequent total and cause-specific mortality and coronary heart disease incidence in men. American Journal of Epidemiology 155, 8 (April 2002), 700-709.

[25] Eletha Flores, Gabriel Tobon, Ettore Cavallaro, Francesca I. Cavallaro, Joel C. Perry, and Thierry Keller. 2008. Improving Patient Motivation in Game Development for Motor Deficit Rehabilitation. In Proceedings of the 2008 International Conference on Advances in Computer Entertainment Technology (ACE '08). ACM, New York, NY, USA, 381-384. https: //doi.org/10.1145/1501750.1501839

[26] Azadeh Forghani, Carman Neustaedter, Manh C. Vu, Tejinder K. Judge, and Alissa N. Antle. 2018. G2G: The Design and Evaluation of a Shared Calendar and Messaging System for Grandparents and Grandchildren. In Proceedings of the 2018 CHI Conference on Human Factors in Computing Systems (CHI '18). ACM, New York, NY, USA, Article 155, 12 pages. https://doi.org/10.1145/3173574.3173729

[27] Jon E. Grahe and Frank J. Bernieri. 1999. The Importance of Nonverbal Cues in Judging Rapport. Journal of Nonverbal Behavior 23, 4 (Dec. 1999), 253-269.

[28] Victoria Groom, Jeremy N. Bailenson, and Clifford Nass. 2009. The influence of racial embodiment on racial bias in immersive virtual environments. Social Influence 4, 3 (2009), 231-248.

[29] Simone Hausknecht, Carman Neustaedter, and David Kaufman. 2017. Blurring the lines of age: intergenerational collaboration in alternate reality games. In Game-Based Learning Across the Lifespan. Springer, 47-64.

[30] James Hodge, Madeline Balaam, Sandra Hastings, and Kellie Morrissey. 2018. Exploring the Design of Tailored Virtual Reality Experiences for People with Dementia. In Proceedings of the 2018 CHI Conference on Human Factors in Computing Systems (CHI '18). ACM, New York, NY, USA, Article 514, 13 pages. https://doi.org/10.1145/3173574.3174088

[31] Alexis Hope, Ted Schwaba, and Anne Marie Piper. 2014. Understanding Digital and Material Social Communications for Older Adults. In Proceedings of the 32nd Annual ACM Conference on Human Factors in Computing Systems (CHI '14). ACM, New York, NY, USA, 3903-3912. https://doi.org/10.1145/2556288.2557133

[32] Aram Kim, Nora Darakjian, and James M. Finley. 2017. Walking in fully immersive virtual environments: an evaluation of potential adverse effects in older adults and individuals with Parkinson's disease. fournal of Neuroengineering and Rehabilitation 14, 1 (2017), 16.

[33] Eugenia M. Kolasinski. 1995. Simulator Sickness in Virtual Environments. Technical Report. Army Research Institute for the Behavioral and Social Sciences, Alexandria VA.

[34] Debbie Laliberte Rudman. 2015. Embodying positive aging and neoliberal rationality: talking about the aging body within narratives of retirement. Journal of Aging Studies 34 (Aug. 2015), 10-20.

[35] Amanda Lazar, Raymundo Cornejo, Caroline Edasis, and Anne Marie Piper. 2016. Designing for the Third Hand: Empowering Older Adults with Cognitive Impairment Through Creating and Sharing. In Proceedings of the 2016 ACM Conference on Designing Interactive Systems (DIS '16). ACM, New York, NY, USA, 1047-1058. https://doi.org/10.1145/ 2901790.2901854 
[36] Amanda Lazar, Hilaire J. Thompson, Shih-Yin Lin, and George Demiris. 2018. Negotiating Relation Work with Telehealth Home Care Companionship Technologies That Support Aging in Place. Proc. ACM Hum.-Comput. Interact. 2, CSCW, Article 103 (Nov. 2018), 19 pages. https://doi.org/10.1145/3274372

[37] Yu-Hao Lee, Min Xiao, and Robert H. Wells. 2018. The Effects of Avatars' Age on Older Adults' Self-Disclosure and Trust. Cyberpsychology, Behavior, and Social Networking 21, 3 (March 2018), 173-178.

[38] June Lennie. 2006. Increasing the rigour and trustworthiness of participatory evaluations: learnings from the field. Evaluation fournal of Australasia 6, 1 (2006), 27-35.

[39] Hao Li, Laura Trutoiu, Kyle Olszewski, Lingyu Wei, Tristan Trutna, Pei-Lun Hsieh, Aaron Nicholls, and Chongyang Ma. 2015. Facial Performance Sensing Head-mounted Display. ACM Transactions on Graphics 34, 4, Article 47 (July 2015), 9 pages. https://doi.org/10.1145/2766939

[40] Ann Light, Tuck W. Leong, and Toni Robertson. 2015. Ageing well with CSCW. In ECSCW 2015: Proceedings of the 14th European Conference on Computer Supported Cooperative Work, 19-23 September 2015, Oslo, Norway. Springer, 295-304.

[41] Siân E. Lindley, Richard Harper, and Abigail Sellen. 2009. Desiring to Be in Touch in a Changing Communications Landscape: Attitudes of Older Adults. In Proceedings of the SIGCHI Conference on Human Factors in Computing Systems (CHI '09). ACM, New York, NY, USA, 1693-1702. https://doi.org/10.1145/1518701.1518962

[42] Yun-e Liu, Ian J. Norman, and Alison E. While. 2013. Nurses' attitudes towards older people: A systematic review. International Journal of Nursing Studies 50, 9 (2013), 1271-1282.

[43] Valeria Manera, Emmanuelle Chapoulie, Jérémy Bourgeois, Rachid Guerchouche, Renaud David, Jan Ondrej, George Drettakis, and Philippe Robert. 2016. A Feasibility Study with Image-Based Rendered Virtual Reality in Patients with Mild Cognitive Impairment and Dementia. PLoS One 11, 3 (March 2016), e0151487.

[44] Victoria McArthur. 2017. The UX of Avatar Customization. In Proceedings of the 2017 CHI Conference on Human Factors in Computing Systems (CHI '17). ACM, New York, NY, USA, 5029-5033. https://doi.org/10.1145/3025453.3026020

[45] Victoria McArthur and Jennifer Jenson. 2014. E is for Everyone? Best Practices for the Socially Inclusive Design of Avatar Creation Interfaces. In Proceedings of the 2014 Conference on Interactive Entertainment (IE2014). ACM, New York, NY, USA, Article 2, 8 pages. https://doi.org/10.1145/2677758.2677783

[46] Victoria McArthur, Robert John Teather, and Jennifer Jenson. 2015. The Avatar Affordances Framework: Mapping Affordances and Design Trends in Character Creation Interfaces. In Proceedings of the 2015 Annual Symposium on Computer-Human Interaction in Play (CHI PLAY '15). ACM, New York, NY, USA, 231-240. https://doi.org/10.1145/ 2793107.2793121

[47] Andrew R. McNeill, Lynne Coventry, Jake Pywell, and Pam Briggs. 2017. Privacy Considerations when Designing Social Network Systems to Support Successful Ageing. In Proceedings of the 2017 CHI Conference on Human Factors in Computing Systems (CHI '17). ACM, New York, NY, USA, 6425-6437. https://doi.org/10.1145/3025453.3025861

[48] Kimberly J. Miller, Brooke S. Adair, Alan J. Pearce, Catherine M. Said, Elizabeth Ozanne, and Meg M. Morris. 2013. Effectiveness and feasibility of virtual reality and gaming system use at home by older adults for enabling physical activity to improve health-related domains: a systematic review. Age and Ageing 43, 2 (2013), 188-195.

[49] Wendy Moyle, Cindy Jones, Toni Dwan, and Tanya Petrovich. 2018. Effectiveness of a Virtual Reality Forest on People With Dementia: A Mixed Methods Pilot Study. Gerontologist 58, 3 (May 2018), 478-487.

[50] Bonnie Nardi and Justin Harris. 2006. Strangers and Friends: Collaborative Play in World of Warcraft. In Proceedings of the 200620 th Anniversary Conference on Computer Supported Cooperative Work (CSCW'06). ACM, New York, NY, USA, 149-158. https://doi.org/10.1145/1180875.1180898

[51] William Lawrence Neuman. 2011. Social research methods : qualitative and quantitative approaches. Allyn \& Bacon, Boston.

[52] Barbara Barbosa Neves, Rachel L. Franz, Cosmin Munteanu, Ronald Baecker, and Mags Ngo. 2015. "My Hand Doesn't Listen to Me!": Adoption and Evaluation of a Communication Technology for the 'Oldest Old'. In Proceedings of the 33rd Annual ACM Conference on Human Factors in Computing Systems (CHI '15). ACM, New York, NY, USA, 1593-1602. https://doi.org/10.1145/2702123.2702430

[53] Kristine Nowak and Jesse Fox. 2018. Avatars and computer-mediated communication: a review of the definitions, uses, and effects of digital representations. Review of Communication Research 6 (2018), 30-53.

[54] Catherine J. O’Brien, Jennifer L. Smith, and Dennis E. Beck. 2016. Real relationships in a virtual world: Social engagement among older adults in Second Life. Gerontechnology 15 (2016), 171-179.

[55] Sofia Adelaide Osimo, Rodrigo Pizarro, Bernhard Spanlang, and Mel Slater. 2015. Conversations between self and self as Sigmund Freud-A virtual body ownership paradigm for self counselling. Scientific Reports 5 (2015), 13899.

[56] Rob Procter, Joe Wherton, Trish Greenhalgh, Paul Sugarhood, Mark Rouncefield, and Sue Hinder. 2016. Telecare call centre work and ageing in place. Computer Supported Cooperative Work (CSCW) 25, 1 (2016), 79-105.

[57] Peter Reason and Hilary Bradbury. 2008. The Sage handbook of action research : participative inquiry and practice. SAGE Publications, Thousand Oaks, California. 
[58] Darren J. Reed and Geraldine Fitzpatrick. 2008. Acting Your Age in Second Life. In International Conference on Fun and Games, Panos Markopoulos, Boris de Ruyter, Wijnand IJsselsteijn, and Duncan Rowland (Eds.). Springer Berlin Heidelberg, Berlin, Heidelberg, 158-169.

[59] Lambèr Royakkers, Jelte Timmer, Linda Kool, and Rinie van Est. 2018. Societal and ethical issues of digitization. Ethics and Information Technology 20, 2 (01 Jun 2018), 127-142. https://doi.org/10.1007/s10676-018-9452-x

[60] Maria T. Schultheis and Albert A. Rizzo. 2001. The application of virtual reality technology in rehabilitation. Rehabilitation Psychology 46, 3 (2001), 296.

[61] Cheryl D. Seals, Wanda Moses, Aqueasha Martin, Kelley Clanton, and Felicia Doswell. 2008. Life Long Learning: Seniors in Second Life Continuum. Journal of Computer Science 4, 12 (2008), 1064-1070.

[62] Sven Seele, Sebastian Misztal, Helmut Buhler, Rainer Herpers, and Jonas Schild. 2017. Here’s Looking At You Anyway!: How Important is Realistic Gaze Behavior in Co-located Social Virtual Reality Games?. In Proceedings of the Annual Symposium on Computer-Human Interaction in Play (CHI PLAY '17). ACM, New York, NY, USA, 531-540. https://doi.org/10.1145/3116595.3116619

[63] Panote Siriaraya and Chee Siang Ang. 2012. Characteristics and usage patterns of older people in a 3D online multi-user virtual environment. Computers in Human Behavior 28, 5 (2012), 1873-1882.

[64] Panote Siriaraya and Chee Siang Ang. 2014. Recreating Living Experiences from Past Memories Through Virtual Worlds for People with Dementia. In Proceedings of the 32nd Annual ACM Conference on Human Factors in Computing Systems (CHI '14). ACM, New York, NY, USA, 3977-3986. https://doi.org/10.1145/2556288.2557035

[65] Panote Siriaraya and Chee Siang Ang. 2019. The Social Interaction Experiences of Older People in a 3D Virtual Environment. In Perspectives on Human-Computer Interaction Research with Older People. Springer, 101-117.

[66] Panote Siriaraya, Chee Siang Ang, and Ania Bobrowicz. 2014. Exploring the potential of virtual worlds in engaging older people and supporting healthy aging. Behaviour \& Information Technology 33, 3 (2014), 283-294.

[67] Nina Skjæret, Ather Nawaz, Tobias Morat, Daniel Schoene, Jorunn Lægdheim Helbostad, and Beatrix Vereijken. 2016. Exercise and rehabilitation delivered through exergames in older adults: an integrative review of technologies, safety and efficacy. International fournal of Medical Informatics 85, 1 (2016), 1-16.

[68] Katsuhiro Suzuki, Fumihiko Nakamura, Jiu Otsuka, Katsutoshi Masai, Yuta Itoh, Yuta Sugiura, and Maki Sugimoto. 2017. Recognition and mapping of facial expressions to avatar by embedded photo reflective sensors in head mounted display. In 2017 IEEE Virtual Reality (VR). IEEE Computer Society, 177-185. https://doi.org/10.1109/VR.2017.7892245

[69] David R. Thomas. 2006. A General Inductive Approach for Analyzing Qualitative Evaluation Data. American fournal of Evaluation 27, 2 (June 2006), 237-246.

[70] Emmanuelle Tulle. 2015. Theorising embodiment and ageing. Handbook of cultural gerontology (2015), 125-133.

[71] David Unbehaun, Daryoush Daniel Vaziri, Konstantin Aal, Rainer Wieching, Peter Tolmie, and Volker Wulf. 2018. Exploring the Potential of Exergames to Affect the Social and Daily Life of People with Dementia and Their Caregivers. In Proceedings of the 2018 CHI Conference on Human Factors in Computing Systems (CHI '18). ACM, New York, NY, USA, Article 62, 15 pages. https://doi.org/10.1145/3173574.3173636

[72] Asimina Vasalou, Adam N. Joinson, and Jeremy Pitt. 2007. Constructing My Online Self: Avatars That Increase Self-focused Attention. In Proceedings of the SIGCHI Conference on Human Factors in Computing Systems (CHI '07). ACM, New York, NY, USA, 445-448. https://doi.org/10.1145/1240624.1240696

[73] Frank Vetere, Hilary Davis, Martin Gibbs, and Steve Howard. 2009. The Magic Box and Collage: Responding to the challenge of distributed intergenerational play. International fournal of Human-Computer Studies 67, 2 (2009), 165-178.

[74] John Vines, Gary Pritchard, Peter Wright, Patrick Olivier, and Katie Brittain. 2015. An Age-Old Problem: Examining the Discourses of Ageing in HCI and Strategies for Future Research. ACM Trans. Comput.-Hum. Interact. 22, 1, Article 2 (Feb. 2015), 27 pages. https://doi.org/10.1145/2696867

[75] Jenny Waycott, Frank Vetere, Sonja Pedell, Lars Kulik, Elizabeth Ozanne, Alan Gruner, and John Downs. 2013. Older Adults As Digital Content Producers. In Proceedings of the SIGCHI Conference on Human Factors in Computing Systems (CHI '13). ACM, New York, NY, USA, 39-48. https://doi.org/10.1145/2470654.2470662

[76] Jenny Waycott, Greg Wadley, Steven Baker, Hasan Shahid Ferdous, Thuong Hoang, Kathrin Gerling, Christopher James Headleand, and Adalberto L. Simeone. 2018. Manipulating Reality?: Designing and Deploying Virtual Reality in Sensitive Settings. In Proceedings of the 2018 ACM Conference Companion Publication on Designing Interactive Systems (DIS '18 Companion). ACM, New York, NY, USA, 411-414. https://doi.org/10.1145/3197391.3197401

[77] G A Wildenbos, Linda Peute, and Monique Jaspers. 2018. Aging barriers influencing mobile health usability for older adults: A literature based framework (MOLD-US). International fournal of Medical Informatics. 114 (June 2018), 66-75. https://doi.org/10.1016/j.ijmedinf.2018.03.012

[78] Alan Yusheng Wu and Cosmin Munteanu. 2018. Understanding Older Users' Acceptance of Wearable Interfaces for Sensor-based Fall Risk Assessment. In Proceedings of the 2018 CHI Conference on Human Factors in Computing Systems (CHI '18). ACM, New York, NY, USA, Article 119, 13 pages. https://doi.org/10.1145/3173574.3173693 
Received April 2019; revised July 2019; accepted September 2019. 